\title{
A comparative study of tree species composition of Panjhora forest beat and Sipchu forest beat of Chalsa forest range, West Bengal, India
}

\author{
Anup Kumar Sarkar*, Manas Dey, Mallika Mazumder \\ Department of Botany, Prasanna Deb Women's College, West Bengal, India.
}

\begin{tabular}{|c|c|}
\hline ARTICLE INFO & ABSTRACT \\
\hline $\begin{array}{l}\text { Article history: } \\
\text { Received on: } 31 / 10 / 2016 \\
\text { Accepted on: } 21 / 12 / 2016 \\
\text { Available online: 20/03/2017 } \\
\text { Key words: } \\
\text { Diversity, Phytosociology, } \\
\text { IVI, Community indices, } \\
\text { Dominance, Codominance. }\end{array}$ & $\begin{array}{l}\text { This study designed to evaluate the ecological diversity of tree vegetation in Panjhora and Sipchu forest beats } \\
\text { of Chalsa forest range, West Bengal, India. Both the forest beats are very well known for their characteristics } \\
\text { as dense forest. To screen the ecological status of these forests a stratified random quadrate method was } \\
\text { employed in the study. A total of } 28 \text { tree species were recorded from Panjhora Forest Beat and } 31 \text { tree species } \\
\text { were recorded from Sipchu Forest Beat. The work conclude that though both the forest beats belong to same } \\
\text { forest range, they differ in pattern of diversity in tree vegetation and specially in regard to the pattern of } \\
\text { dominance. }\end{array}$ \\
\hline
\end{tabular}

\section{INTRODUCTION}

Forests are the principal bio-resources and repositories of natural wealth that support human well-being and ecological sustainability. The forest ecosystems provide unequal share to the world's biodiversity [1]. Thus for the maintenance of biodiversity it is essential to attain forest sustainability [12]. It is undoubtedly justified that the long term sustainability of forest ecosystems is greatly concerned with plant diversity and their phytosociological attributes. Plant diversity is widely acknowledged to support many other communities of forests as well as human community. Much of the overall diversity depends on plant diversity, because plants provide both food and habitat for other organisms [6]. The ecological security of any country depends on the health of its forests [7]. Thus management and maintenance of any forest is obligatory. As the over al condition of forest depends on its plant composition, the information on composition, diversity and ecological aspects of plant species is of primary importance in the planning and implementation of forest biodiversity conservation efforts. In a typical forest knowledge of vascular plant diversity

\footnotetext{
* Corresponding Author

Anup Kumar Sarkar, Department Of Botany, Prasanna Deb Women's

College,West Bengal, India.E-mail:anupsarkar.jpg@gmail.com
}

and changes that occur with disturbance may provide planning information to Biologists [16]. Among the vascular plants tree species are much important as they are controlling the keystone factor, i.e., entry of light in to forest bed. Analysis and estimation of Tree diversity, in which a combination of physical habitat, vegetation, physiognomy, species composition and community relationship are useful in formulating forest management programme [15]. The inherent variation within communities and ecosystems must be documented and used for base-line data to effectively predict the outcome of disturbances, such as regeneration harvest methods, on floristic diversity and richness [15].

\section{MATERIAL AND METHOD}

\subsection{Description of Study Site}

The present study has been carried out in two beat forests of Chalsa Forest Range of Jalpaiguri Forest Division, West Bengal, India, namely Panjhora Beat and Sipchu Beat. Both the forest beats are located on the undulating plain of Himalayan foothill, which create a great floral and faunal diversity. It is located in close proximity to Chapramari Wildlife Sanctuay. The forest is situated very close to the bank of the Jaldhaka and Murti River. Sipchu forest Beat covers $1757.58 \mathrm{Ha}$ area and Panjhora forest beat Covers 2255.92 Ha. These forests are characterized by the presence of natural water passages, called 'Jhora'. 


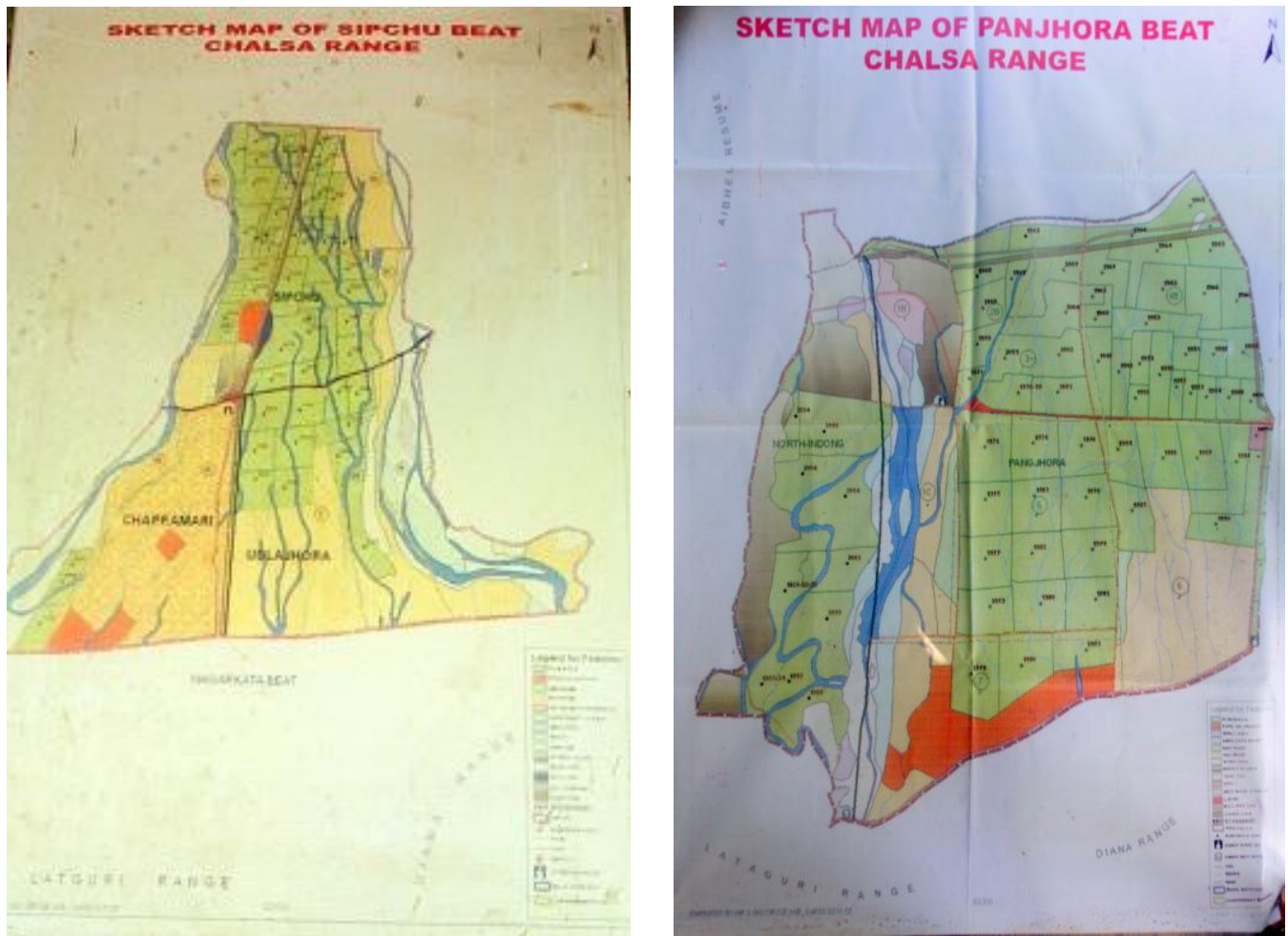

Fig 1: Sketch Map Of Sipchu Forest Beat and Panjhora Forest Beat

\subsection{Study of Tree vegetation composition and structure}

For phytosociological studies of tree vegetation in the selected beat forest of Chalsa forest range, the quadrate methods were used. In each beat forest, a total of 20 sampling sites representing various categories of natural forests and plantations were selected for vegetation sampling. At each sampling site four quadrates $(20 \mathrm{~m} \times 20 \mathrm{~m})$ were laid to quantify various tree vegetation. The use of local name of each forest site was adopted from the knowledge of Forest guards. Different topography and altitudes, had different types and levels of disturbance intensity; and the dominant and character species for each of the twenty forest community sites were different. Tree species found within each sampling plot were photographed and identified by their vernacular names (adopted from Range Officer, Beat Officer, Forest Guards and local people) and scientific names using various books, articles [5;18] and internet. In order to analyze the diversity of tree vegetation Frequency, Relative frequency, density and Relative density were calculated. Importance Value Index was calculated by adding Relative frequency Relative density and Relative Basal Aea [3, 13].

\section{(a) Frequency (\%)}

This term refers to the degree of dispersion of individual species in an area and usually expressed in terms of percentage. It is calculated by the equation:

$$
\text { Frequency }(\%)=\frac{\text { No. of plot in which the species is present }}{\text { Total No. of plots sampled }} \times 100
$$

\section{(b) Relative Frequency (\%)}

The degree of dispersion of individual species in an area in relation to the number of all the species occurred.

$$
\text { Relative Frequency }(\%)=\frac{\text { Frequency of the species }}{\text { Frequency of all the species }} \times 100
$$

\section{(c) Density}

Density is an expression of the numerical strength of a species where the total number of individuals of each species in all the quadrats is divided by the total number of quadrats studied. Density is calculated by the equation:

$$
\text { Density }=\frac{\text { No. individuals of the species }}{\text { Total No. of plots sampled }}
$$

\section{(d) Relative Density (\%)}

Relative density is the study of numerical strength of a species in relation to the total number of individuals of all the species and can be calculated as:

$$
\text { Relative Density }=\frac{\text { Density of the species }}{\text { Density of all the species }} \times 100
$$

\section{(e) Relative Dominance (\%)}

Dominance of a species is determined by the value of the basal area. Relative dominance is the coverage value of a species with respect to the sum of coverage of the rest of the species in the area. 


$$
\text { Basal Area }=\frac{\text { (Circumference at breast height }) 2}{12.56}
$$

Relative dominance or Relative Basala Area

$$
=\frac{\text { Basal Area of the species }}{\text { Basal Area of all the species }} \times 100
$$

\section{(f) Abundance}

It is the study of the number of individuals of different species in the community per unit area. By quadrats method, samplings are made at random at several places and the number of individuals of each species was summed up for all the quadrats divided by the total number of quadrats in which the species occurred. It is represented by the equation:

$$
\text { Abundance }=\frac{\text { No. individuals of the species }}{\text { Total No. of plots in which the species is present }}
$$

\section{(g) Importance Value Index}

This index is used to determine the overall importance of each species in the community structure. In calculating this index, the percentage values of the relative frequency, relative density and relative dominance (Relative Basala Area) are summed up together and this value is designated as the Importance Value Index or IVI of the species.

IVI= Relative Frequency + Relative Density + Relative dominance

\subsection{Data processing and Phytosociological Analysis:}

All the data both spatial and especial collected from different sources has been tabulated and analyzed separately. The data collected were used to compute community indices like

\section{(a) Species diversity $\left(\mathbf{H}^{\prime}\right)$}

Species diversity of different tree species; it was calculated using the Shannon- Weiner Index: (Shannon and Weiner, 1963).

$$
\left(\mathrm{H}^{\prime}\right)=-\sum[(\mathrm{ni} / \mathrm{N}) \cdot \ln (\mathrm{ni} / \mathrm{N})]
$$

Where 'ni' is the IVI of individual species and $\mathrm{N}$ is the total IVI of all the species [17].

\section{(b) Species dominance (Cd)}

Species dominance was calculated following Simpson:

$$
\mathrm{Cd}=\Sigma(\mathrm{ni} / \mathrm{N})^{2} \text {, }
$$

where, ni and $\mathrm{N}$ are the same as those for Shannon Weiner information function [18].

\section{(c) Equitability of evenness (e)}

Equitability of evenness refers to the degree of relative dominance of each species in that area. It was calculated as: Evenness $(\mathrm{e})=\mathrm{H}^{\prime} / \log \mathrm{S}$

where, $H^{\prime}=$ Shannon index, $S=$ number of species [14]. (d) Species richness (D)

Species richness was determined by Margalef index (1968) as:

$$
\mathrm{D}=(\mathrm{S}-1) / \ln \mathrm{N} \text {. }
$$

where, $\mathrm{S}=$ number of species.

$\mathrm{N}=$ total number of individuals [10].

(e)Menhinick's index $\left(\mathrm{D}_{\mathrm{mm}}\right)$

Menhinick's index (Whittaker, 1977). is expressed as $\mathrm{D}_{\mathrm{mm}}=\mathrm{S} / \mathrm{N}$, where $\mathrm{N}$ is the number of individuals in the sample and $\mathrm{S}$ is the species number [11].

\section{(f) Equitability Index}

The Shannon's equitability Index (Lloyd and Ghelard, 1964) is expressed as

$$
(\mathrm{EH})=\mathrm{H} / \mathrm{Hmax}=\mathrm{H} / \mathrm{ln} \mathrm{S} .
$$

Where, $\mathrm{H}$ is the Shannon index and $\mathrm{S}$ is the species number [9].

\section{(g) Berger-Parker Dominance Index}

The Berger-Parker Dominance Index is a simple measure of the numerical importance of the most abundant species and is expressed a $\mathrm{d}=\mathrm{Nmax} / \mathrm{N}$.

$\mathrm{N}_{\text {max }}$ is the number of individuals in the most abundant species and $\mathrm{N}$ is the total number of individuals in the sample. The increase in the value of reciprocal of Berger-Parker Dominance Index reflects the increase in diversity and a reduction in dominance [2].

\section{RESULTS AND DISCUSSION}

The present study showed that both the forest beats are rich in tree diversity. A total of 28 tree species were recorded from Panjhora Forest Beat. Among them highest IVI was recorded for Shorea robusta Gaertn.(15.483). IVI was also good for Schima wallichii Choisy. (10.538) and Wrightia tinctoria (Roxb.) R.Br (9.562). The lowest IVI was recorded for Ailanthus grandis Prain (0.950). IVI was also poor for few species like Dillenia indica L., Gynocardia odorata R. Br., Alstonia scholaris R. Br., Butea monosperma (Lam.) Taub., Beilschmiedia roxburghiana Nees, Toona ciliata M.Roem., Altingia excelsa Noronha. (Table 1). From Sipchu Beat Forest 31 tree species were recorded. Among the tree species highest IVI was recorded for Beilschmiedia roxburghiana Nees (43.867).It was also observed that a few species including Shorea robusta Gaertn., Tetrameles nudiflora R.Br., Sterculia villosa Roxb., Wrightia tomentosa Roem. \& Schult., Stereospermum tetragonum DC., Persea fructifera Kosterm had quite good IVI (Table 2). The lowest IVI was recorded for Andromeda elliptica Siebold \& Zucc. (1.015). 
Table 1: Different Phytosociological values of tree vegetation of Panjhora Forest Beat.

\begin{tabular}{|c|c|c|c|c|c|c|c|c|c|c|}
\hline Sl. No. & Name of The Plant & Family & $\mathbf{A}$ & D & $\mathbf{F r}$ & BA & RD & $\mathbf{R F}$ & RBA & IVI \\
\hline 1 & Terminalia belerica Roxb. & Combretaceae & 3.00 & 0.60 & 20 & 1099.22 & 0.038 & 3.773 & 0.024 & 3.835 \\
\hline 2 & Terminalia alata Roth. & Combretaceae & 2.40 & 0.60 & 25 & 1198.67 & 0.038 & 4.717 & 0.026 & 4.781 \\
\hline 3 & Dillenia indica $\mathrm{L}$. & Dilleniaceae & 2.00 & 0.10 & 5 & 395.72 & 0.006 & 0.943 & 0.009 & 0.958 \\
\hline 4 & Alstonia scholaris $\mathrm{R}$. Br. & Apocynaceae & 1.00 & 0.05 & 5 & 703.50 & 0.003 & 0.943 & 0.015 & 0.961 \\
\hline 5 & Amoora rohituka W. \& A. & Meliaceae & 2.75 & 0.55 & 20 & 1803.84 & 0.035 & 3.773 & 0.039 & 3.847 \\
\hline 6 & Amoora spectabilis Miq. & Meliaceae & 2.40 & 0.60 & 25 & 983.62 & 0.038 & 4.717 & 0.022 & 4.777 \\
\hline 7 & Schima wallichii Choisy. & Theaceae & 3.90 & 2.15 & 55 & 1096.79 & 0.137 & 10.377 & 0.024 & 10.538 \\
\hline 8 & Shorea robusta Gaertn. & Dipterocarpaceae & 5.44 & 4.35 & 80 & 5155.65 & 0.276 & 15.094 & 0.113 & 15.483 \\
\hline 9 & Gynocardia odorata R.Br. & Achariaceae & 1.00 & 0.05 & 5 & 659.32 & 0.003 & 0.943 & 0.014 & 0.960 \\
\hline 10 & Castanopsis tribuloides A. DC. & Fagaceae & 2.17 & 0.65 & 30 & 2942.07 & 0.041 & 5.660 & 0.065 & 5.766 \\
\hline 11 & Wrightia tinctoria (Roxb.) R. Br & Apocynaceae & 4.00 & 2.00 & 50 & 51.33 & 0.127 & 9.434 & 0.001 & 9.562 \\
\hline 12 & Heteropanax fragrans(Roxb.) Seem. & Araliaceae & 1.00 & 0.10 & 10 & 42.12 & 0.006 & 1.887 & 0.001 & 1.894 \\
\hline 13 & Wrightia arborea (Dennst.) Mabb., & Apocynaceae & 2.00 & 0.20 & 10 & 2445.27 & 0.013 & 1.887 & 0.054 & 1.954 \\
\hline 14 & Lagerstromia speciosa Pers. & Lythraceae & 2.80 & 0.70 & 25 & 986.99 & 0.044 & 4.717 & 0.022 & 4.783 \\
\hline 15 & Sterculia villosa Roxb & Sterculiaceae & 2.50 & 0.25 & 10 & 3046.13 & 0.016 & 1.887 & 0.067 & 1.970 \\
\hline 16 & Tetrameles nudiflora $\mathrm{R} . \mathrm{Br}$. & Datiscaceae & 1.00 & 0.10 & 10 & 5804.14 & 0.006 & 1.887 & 0.127 & 2.020 \\
\hline 17 & Butea monosperma (Lam.) Taub. & Fabaceae & 1.00 & 0.05 & 5 & 894.59 & 0.003 & 0.943 & 0.020 & 0.966 \\
\hline 18 & Acrocarpus fraxinifolius Arn. & Fabaceae & 2.17 & 0.65 & 30 & 612.22 & 0.041 & 5.660 & 0.013 & 5.714 \\
\hline 19 & Stereospermum tetragonum DC. & Bignoniaceae & 2.80 & 0.70 & 25 & 1503.74 & 0.044 & 4.717 & 0.033 & 4.794 \\
\hline 20 & Lagerstroemia parviflora Roxb. & Lythraceae & 2.67 & 0.40 & 15 & 1141.72 & 0.025 & 2.830 & 0.025 & 2.880 \\
\hline 21 & Premna mисronata Roxb. & Verbenaceae & 3.00 & 0.45 & 15 & 1281.93 & 0.029 & 2.830 & 0.028 & 2.887 \\
\hline 22 & Beilschmiedia roxburghiana Nees & Lauraceae & 2.00 & 0.10 & 5 & 1827.41 & 0.006 & 0.943 & 0.040 & 0.989 \\
\hline 23 & Toona ciliata M.Roem. & Meliaceae & 1.00 & 0.05 & 5 & 1264.01 & 0.003 & 0.943 & 0.028 & 0.974 \\
\hline 24 & Dillenia pentagyna Roxb. & Dilleniaceae & 2.00 & 0.20 & 10 & 1560.51 & 0.013 & 1.887 & 0.034 & 1.934 \\
\hline 25 & Syzygium cumini (Linn.) Skeels & Myrtaceae & 2.00 & 0.50 & 25 & 3266.76 & 0.032 & 4.717 & 0.072 & 4.821 \\
\hline 26 & Ailanthus grandis Prain & Simaroubaceae & 2.00 & 0.10 & 5 & 27.25 & 0.006 & 0.943 & 0.001 & 0.950 \\
\hline 27 & Altingia excelsa Noronha & Hamamelidaceae & 2.00 & 0.10 & 5 & 886.17 & 0.006 & 0.943 & 0.019 & 0.968 \\
\hline 28 & Casearia graveolens Dalz. & Flacourtiaceae & 1.00 & 0.05 & 5 & 2874.20 & 0.003 & 0.943 & 0.063 & 1.009 \\
\hline
\end{tabular}

$\mathrm{A}=$ Abundance, $\mathrm{D}=$ Density, Fr= Frequency, $\mathrm{BA}=$ Basal Area, RD=Relative Density, $\mathrm{RF}=$ Relative Frequency, RBA= Relative Basal Area, IVI= Importance Value Index

Table 2: Different Phytosociological values of tree vegetation of Sipchu Forest Beat.

\begin{tabular}{|c|c|c|c|c|c|c|c|c|c|c|}
\hline SI & Name Of The Plant & Family & $\mathbf{A}$ & D & $\mathbf{F}$ & B A & RD & RF & RBA & IVI \\
\hline 1. & Amoora rohituka W. \& A. & Meliaceae & 2.50 & 0.50 & 10 & 1678.58 & 3.24 & 1.388 & 1.728 & 6.356 \\
\hline 2. & Acrocarpus fraxinifolius Wight \&Schult & Fabaceae & 2.00 & 0.20 & 10 & 2108.88 & 1.29 & 1.388 & 2.172 & 4.850 \\
\hline 3. & Shorea robusta Gaertn. & Dipterocarpaceae & 2.45 & 1.35 & 55 & 18656.59 & 8.76 & 7.638 & 19.21 & 35.608 \\
\hline 4. & Terminalia belerica Roxb. & Combretaceae & 2.33 & 0.70 & 30 & 1747.24 & 4.65 & 4.166 & 1.799 & 10.615 \\
\hline 5. & Elaeocarpus lanceaefolium Roxb. & Elaeocarpaceae & 2.50 & 0.50 & 20 & 9.75 & 3.24 & 2.777 & 0.010 & 6.027 \\
\hline 6. & Duabanga sonneratioides Buch.-Ham. & Lythraceae & 2.00 & 0.30 & 15 & 1380.12 & 1.94 & 2.083 & 1.421 & 5.444 \\
\hline 7. & Castanopsis tribuloides A. DC. & Fagaceae & 2.50 & 0.25 & 10 & 725.37 & 1.62 & 1.388 & 0.747 & 3.755 \\
\hline 8. & Trewia nudiflora $\mathrm{L}$. & Euphorbiaceae & 1.66 & 0.25 & 15 & 2579.61 & 1.62 & 2.083 & 2.656 & 6.359 \\
\hline 9. & Andromeda elliptica Siebold \& Zucc. & Ericaceae & 1.00 & 0.05 & 05 & 0.71 & 0.32 & 0.694 & 0.001 & 1.015 \\
\hline 10. & Mallotus philippensis (Lam.) Muell.-Arg & Euphorbiaceae & 1.00 & 0.05 & 05 & 121.09 & 0.32 & 0.694 & 0.124 & 1.138 \\
\hline 11. & Schleichera oleosa (Lou.) Oken. & Sapindaceae & 2.00 & 0.20 & 10 & 43.96 & 1.29 & 1.388 & 0.045 & 2.723 \\
\hline 12. & Schima wallichii Choisy. & Theaceae & 1.92 & 1.25 & 65 & 731.77 & 8.11 & 9.027 & 0.753 & 17.89 \\
\hline 13. & Bauhinia triandra Roxb & Fabaceae & 2.00 & 0.30 & 15 & 977.96 & 1.94 & 2.083 & 1.007 & 5.030 \\
\hline 14. & Stereospermum tetragonum DC. & Bignoniaceae & 2.37 & 0.95 & 40 & 1780.90 & 6.16 & 5.555 & 1.834 & 13.549 \\
\hline 15. & Beilschmiedia roxburghiana Nees & Lauraceae & 2.64 & 2.25 & 85 & 16949.46 & 14.61 & 11.80 & 17.457 & 43.867 \\
\hline 16. & Tetrameles nudiflora R.Br. & Datiscaceae & 1.87 & 0.75 & 40 & 16693.66 & 4.87 & 5.555 & 17.193 & 27.618 \\
\hline 17. & Sterculia villosa Roxb. & Sterculiaceae & 1.75 & 1.05 & 60 & 5165.38 & 6.81 & 8.333 & 5.320 & 20.463 \\
\hline 18. & Wrightia tomentosa Roem. \& Schult. & Apocynaceae & 2.90 & 1.60 & 55 & 98.03 & 10.38 & 7.638 & 0.100 & 18.118 \\
\hline 19. & Altingia excelsa Noronha & Hamamelidaceae & 2.16 & 0.15 & 30 & 567.54 & 0.97 & 2.083 & 0.584 & 3.637 \\
\hline 20. & Polyalthia simiarum Benth. & Anonaceae & 2.50 & 0.25 & 10 & 1529.45 & 1.62 & 1.388 & 1.574 & 4.582 \\
\hline 21. & Premna mucronata Roxb. & Verbenaceae & 2.00 & 0.30 & 15 & 1096.04 & 1.94 & 2.083 & 1.128 & 5.151 \\
\hline 22. & Bischofia javanica Blume & Phyllanthaceae & 2.00 & 0.10 & 05 & 1156.07 & 0.64 & 0.694 & 1.190 & 2.524 \\
\hline 23. & Macaranga indica Gagnep. & Euphorbiaceae & 2.00 & 0.20 & 10 & 812.18 & 1.29 & 1.388 & 0.836 & 3.514 \\
\hline 24. & Terminalia alata Roth. & Combretaceae & 3.00 & 0.05 & 05 & 1311.19 & 0.32 & 0.694 & 1.350 & 2.364 \\
\hline 25. & Ailanthus grandis Prain & Simaroubaceae & 2.00 & 0.20 & 10 & 293.83 & 1.29 & 1.388 & 0.302 & 2.980 \\
\hline 26. & Chuckrasia tabularis A. Juss. & Meliaceae & 2.50 & 0.25 & 10 & 308.02 & 1.62 & 1.388 & 0.317 & 3.325 \\
\hline 27. & Camphorina cassia (L.) Farw & Lauraceae & 1.50 & 0.15 & 10 & 40.18 & 0.97 & 1.388 & 0.041 & 2.399 \\
\hline 28. & Persea fructifera Kosterm & Lauraceae & 2.00 & 0.20 & 10 & 13075.44 & 1.29 & 1.388 & 13.467 & 16.145 \\
\hline 29. & Careya arborea Roxb. & Lecythidaceae & 2.00 & 0.70 & 35 & 2040.00 & 4.54 & 4.861 & 2.101 & 11.502 \\
\hline 30. & Lagerstroemia parviflora Roxb. & Lythraceae & 1.50 & 0.15 & 10 & 501.05 & 0.97 & 1.388 & 0.516 & 2.874 \\
\hline 31. & Dillenia pentagyna Roxb. & Dilleniaceae & 1.33 & 0.20 & 15 & 2912.14 & 1.29 & 2.083 & 2.999 & 6.372 \\
\hline
\end{tabular}


Table 3: Different index values of tree vegetation of Panjhora Forest Beat.

\begin{tabular}{|c|c|c|c|c|}
\hline Sl. No. & Name of The Plant & Shannon Index & Species dominance & Evenness \\
\hline 1 & Terminalia belerica Roxb. & 0.12253 & 0.00138 & 0.08467 \\
\hline 2 & Terminalia alata Roth. & 0.14252 & 0.00215 & 0.98491 \\
\hline 3 & Dillenia indica $\mathrm{L}$. & 0.04330 & 0.00008 & 0.02992 \\
\hline 4 & Alstonia scholaris $\mathrm{R} . \mathrm{Br}$. & 0.04362 & 0.00008 & 0.03014 \\
\hline 5 & Amoora rohituka W. \& A. & 0.12280 & 0.00139 & 0.08486 \\
\hline 6 & Amoora spectabilis Miq. & 0.14242 & 0.00215 & 0.09842 \\
\hline 7 & Schima wallichii Choisy. & 0.23327 & 0.01047 & 0.16120 \\
\hline 8 & Shorea robusta Gaertn. & 0.28488 & 0.02260 & 0.19686 \\
\hline 9 & Gynocardia odorata R.Br. & 0.04379 & 0.00008 & 0.03026 \\
\hline 10 & Castanopsis tribuloides A. DC. & 0.16140 & 0.00313 & 0.10823 \\
\hline 11 & Wrightia tinctoria (Roxb.) R. Br & 0.22069 & 0.00862 & 0.15250 \\
\hline 12 & Heteropanax fragrans (Roxb.) Seem. & 0.07348 & 0.00033 & 0.05078 \\
\hline 13 & Wrightia arborea (Dennst.) Mabb., & 0.00752 & 0.00036 & 0.05198 \\
\hline 14 & Lagerstromia speciosa Pers. & 0.14256 & 0.00211 & 0.09852 \\
\hline 15 & Sterculia villosa Roxb & 0.07569 & 0.00572 & 0.05230 \\
\hline 16 & Tetrameles nudiflora $\mathrm{R} . \mathrm{Br}$. & 0.07711 & 0.00038 & 0.05328 \\
\hline 17 & Butea monosperma (Lam.) Taub. & 0.04380 & 0.00008 & 0.03026 \\
\hline 18 & Acrocarpus fraxinifolius Arn. & 0.16045 & 0.00307 & 0.11087 \\
\hline 19 & Stereospermum tetragonum DC. & 0.14278 & 0.00216 & 0.09867 \\
\hline 20 & Lagerstroemia parviflora Roxb. & 0.10003 & 0.00078 & 0.06912 \\
\hline 21 & Premna mисronata Roxb. & 0.10020 & 0.00076 & 0.06924 \\
\hline 22 & Beilschmiedia roxburghiana Nees & 0.04461 & 0.00009 & 0.03083 \\
\hline 23 & Toona ciliata M.Roem. & 0.04408 & 0.00008 & 0.03109 \\
\hline 24 & Dillenia pentagyna Roxb. & 0.07465 & 0.00035 & 0.05158 \\
\hline 25 & Syzygium cumini (Linn.) Skeels & 0.14332 & 0.00219 & 0.09904 \\
\hline 26 & Ailanthus grandis Prain & 0.04322 & 0.00008 & 0.02991 \\
\hline 27 & Altingia excelsa Noronha & 0.04387 & 0.00009 & 0.03031 \\
\hline 28 & Casearia graveolens Dalz. & 0.04532 & 0.00009 & 0.03131 \\
\hline
\end{tabular}

Table 4: Different index values of tree vegetation of Sipchu Forest Beat.

\begin{tabular}{|c|c|c|c|c|}
\hline Sl. No. & Name of The Plant & Shannon Index & Species dominance & Evenness \\
\hline 1 & Amoora rohituka W. \& A. & 0.08198 & 0.00045 & 0.05444 \\
\hline 2 & Acrocarpus fraxinifolius Wight $\&$ Schult. & 0.06700 & 0.00026 & 0.04497 \\
\hline 3 & Shorea robusta Gaertn. & 0.25386 & 0.01428 & 0.17022 \\
\hline 4 & Terminalia belerica Roxb. & 0.11878 & 0.00126 & 0.07964 \\
\hline 5 & Elaeocarpus lanceaefolium Roxb. & 0.07887 & 0.00040 & 0.05288 \\
\hline 6 & Duabanga sonneratioides Buch.-Ham. & 0.07312 & 0.00033 & 0.04903 \\
\hline 7 & Castanopsis tribuloides A. DC. & 0.05513 & 0.00015 & 0.03695 \\
\hline 8 & Trewia nudiflora $\mathrm{L}$. & 0.08209 & 0.00045 & 0.05504 \\
\hline 9 & Andromeda elliptica Siebold \& Zucc. & 0.01935 & 0.00001 & 0.01297 \\
\hline 10 & Mallotus philippensis (Lam.) Muell.-Arg & 0.02122 & 0.00001 & 0.01422 \\
\hline 11 & Schleichera oleosa (Lou.) Oken. & 0.04291 & 0.00008 & 0.02877 \\
\hline 12 & Schima wallichii Choisy. & 0.16887 & 0.00360 & 0.11323 \\
\hline 13 & Bauhinia triandra Roxb & 0.06889 & 0.00028 & 0.04619 \\
\hline 14 & Stereospermum tetragonum DC. & 0.14038 & 0.00206 & 0.09413 \\
\hline 15 & Beilschmiedia roxburghiana Nees & 0.28205 & 0.02167 & 0.18913 \\
\hline 16 & Tetrameles nudiflora $\mathrm{R} . \mathrm{Br}$. & 0.22046 & 0.00859 & 0.14779 \\
\hline 17 & Sterculia villosa Roxb. & 0.18394 & 0.00471 & 0.12334 \\
\hline 18 & Wrightia tomentosa Roem. \& Schult. & 0.17026 & 0.00369 & 0.11417 \\
\hline 19 & Altingia excelsa Noronha & 0.05375 & 0.00014 & 0.03604 \\
\hline 20 & Polyalthia simiarum Benth. & 0.06420 & 0.00023 & 0.04304 \\
\hline 21 & Premna mucronata Roxb. & 0.07015 & 0.00029 & 0.04704 \\
\hline 22 & Bischofia javanica Blume & 0.00404 & 0.00007 & 0.02709 \\
\hline 23 & Macaranga indica Gagnep. & 0.05236 & 0.00013 & 0.03511 \\
\hline 24 & Terminalia alata Roth. & 0.03837 & 0.00006 & 0.02573 \\
\hline 25 & Ailanthus grandis Prain & 0.04604 & 0.00010 & 0.03087 \\
\hline 26 & Chuckrasia tabularis A. Juss. & 0.05011 & 0.00012 & 0.03607 \\
\hline 27 & Camphorina cassia (L.) Farw & 0.03881 & 0.00006 & 0.02602 \\
\hline 28 & Persea fructifera Kosterm & 0.15797 & 0.00293 & 0.10592 \\
\hline 29 & Careya arborea Roxb. & 0.12563 & 0.00149 & 0.08424 \\
\hline 30 & Lagerstroemia parviflora Roxb. & 0.04476 & 0.00009 & 0.03002 \\
\hline 31 & Dillenia pentagyna Roxb. & 0.08220 & 0.00045 & 0.05511 \\
\hline
\end{tabular}




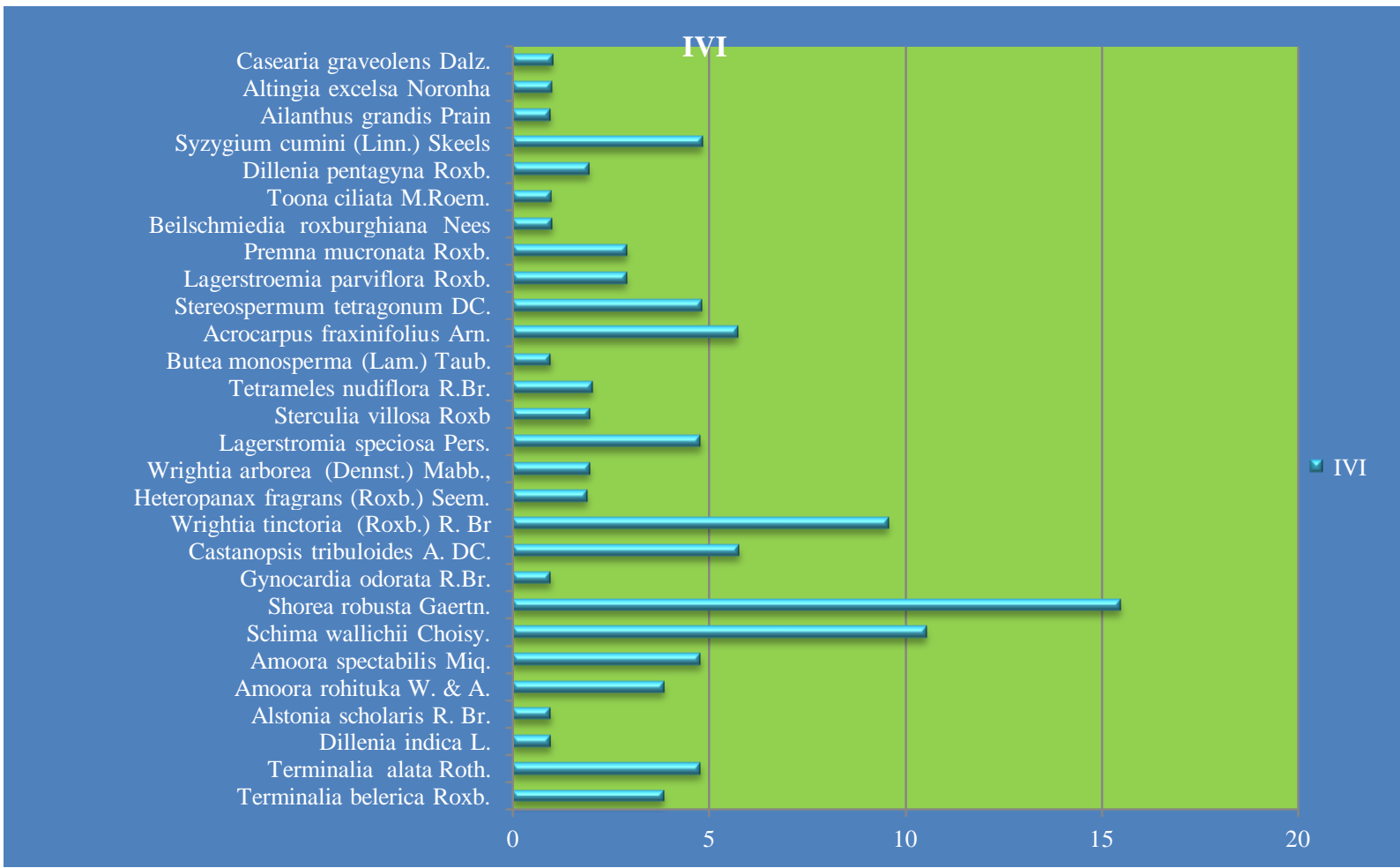

Graph 1: IVI of tree species in Panjhora beat forest

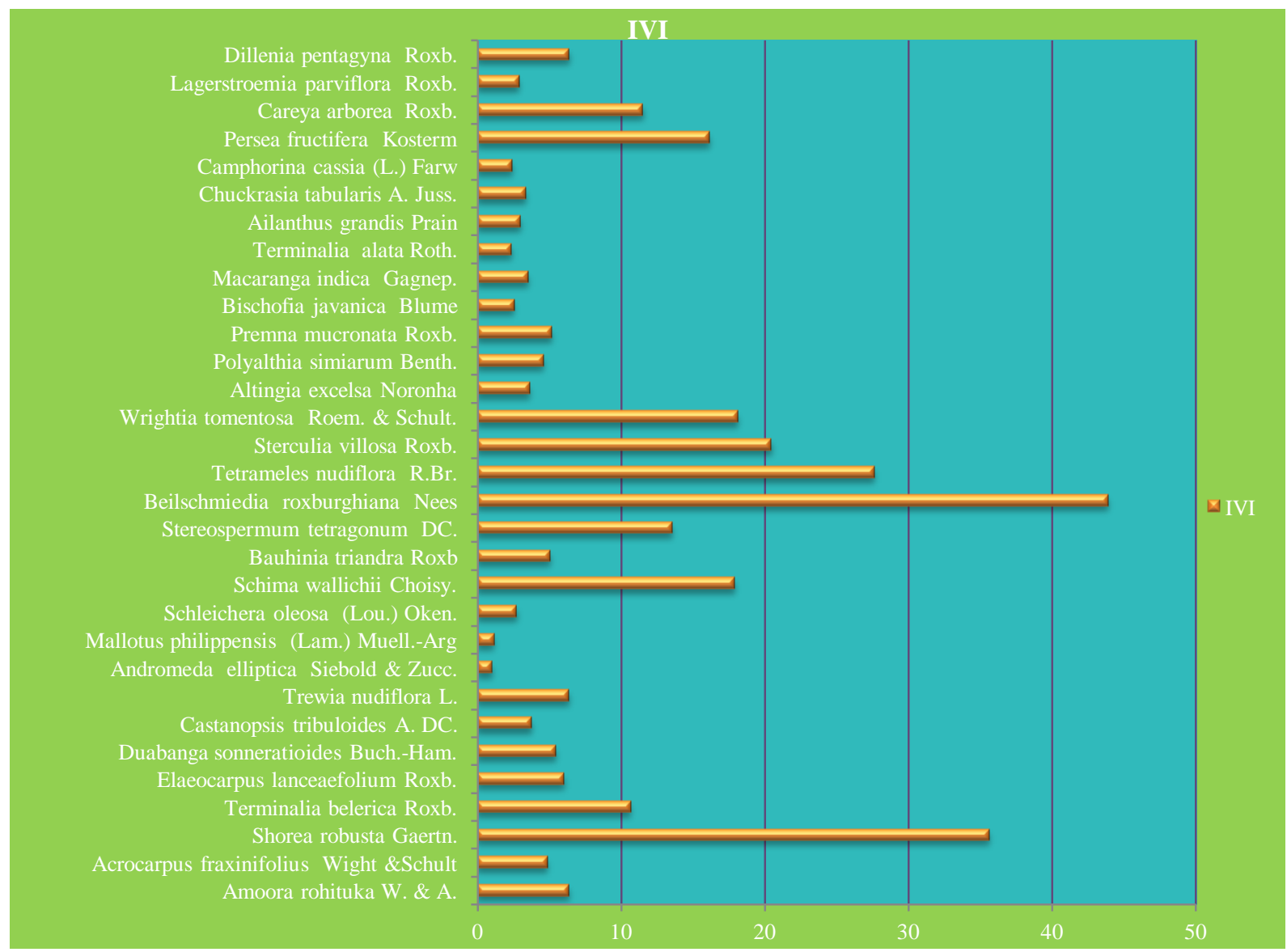

Graph.2: IVI of tree species in Sipchu beat forest 


\section{CONCLUSION}

The paper reflects the phytosociological and ecological characters of tree vegetation in to two beat forests namely Panjhora Beat and Sipchu Beat of Chalsa range area in Jalpaiguri forest division, West Bengal, India. The structural composition of flora found in these beat forests were quite different. The differences were also noticed in the composition of tree vegetation. In Panjhora beat Shorea robusta Gaertn. was found as the dominant species and it had quite higher IVI then the other tree species. Where as in Sipchu beat Beilschmiedia roxburghiana Nees was found as the dominant species. But in Sipchu few species had nearest IVI in respect to dominant species. Among them Shorea robusta Gaertn. had very close IVI in regard to Beilschmiedia roxburghiana Nees. Here the Species diversity index value of tree species at Panjhora Beat was found as 2.8785 and Species richness was 5.5725 . Where as the Species diversity index value of tree species at Sipchu Beat was found as 2.9575 and Species richness was 5.1980. Both the indices showed that slightly high tree species diversity was accounted in Sipchu Beat than Panjhora Beat. It is important to note that high diversity status in terms of all the above indices was reflected in Sipchu Beat.The evenness indices showed that there was a very little difference in evenness in between the forest beats. The Margalef's and Menhinick's indices also revealed the similar trend like that of evenness index. There was also a significant diference in BergerParker Dominance Index. The indeces revealed that Panjhora beat had comparatively low diversity in tree vegetation and high level of Dominance. On the contrary Sipchu beat had high diversity and low level of dominance among tree species. More than one tree species of Sipchu beat showed good IVI and thus it was concluded that the tree vegetation of Siphu beat support the concept of ecological codominance. The study suggested to the followers for the study of soil seed bank and allelopathic interactions among the tree species as well as other plants and microbes in both the forest beats. It is also suggested for the study of successive pattern of the plant communities in these forest beats.

Table 5: Different Community indices of tree vegetation of the Forest Beats

\begin{tabular}{ccc}
\hline Community indices Value & Panjhora Beat & Sipchu Beat \\
\hline Species diversity (H') & 2.8785 & 2.9575 \\
Species dominance (Cd) & 0.0707 & 0.0684 \\
Equitability of evenness (e) & 1.9893 & 1.9831 \\
Species richness (d) & 5.5725 & 5.1980 \\
Menhinick's index (D $\left.\mathrm{D}_{\mathrm{mm}}\right)$ & 0.0828 & 0.0965 \\
Equitability Index & 0.8734 & 0.8612 \\
Berger-Parker Dominance Index & 0.2668 & 0.1401 \\
\hline
\end{tabular}

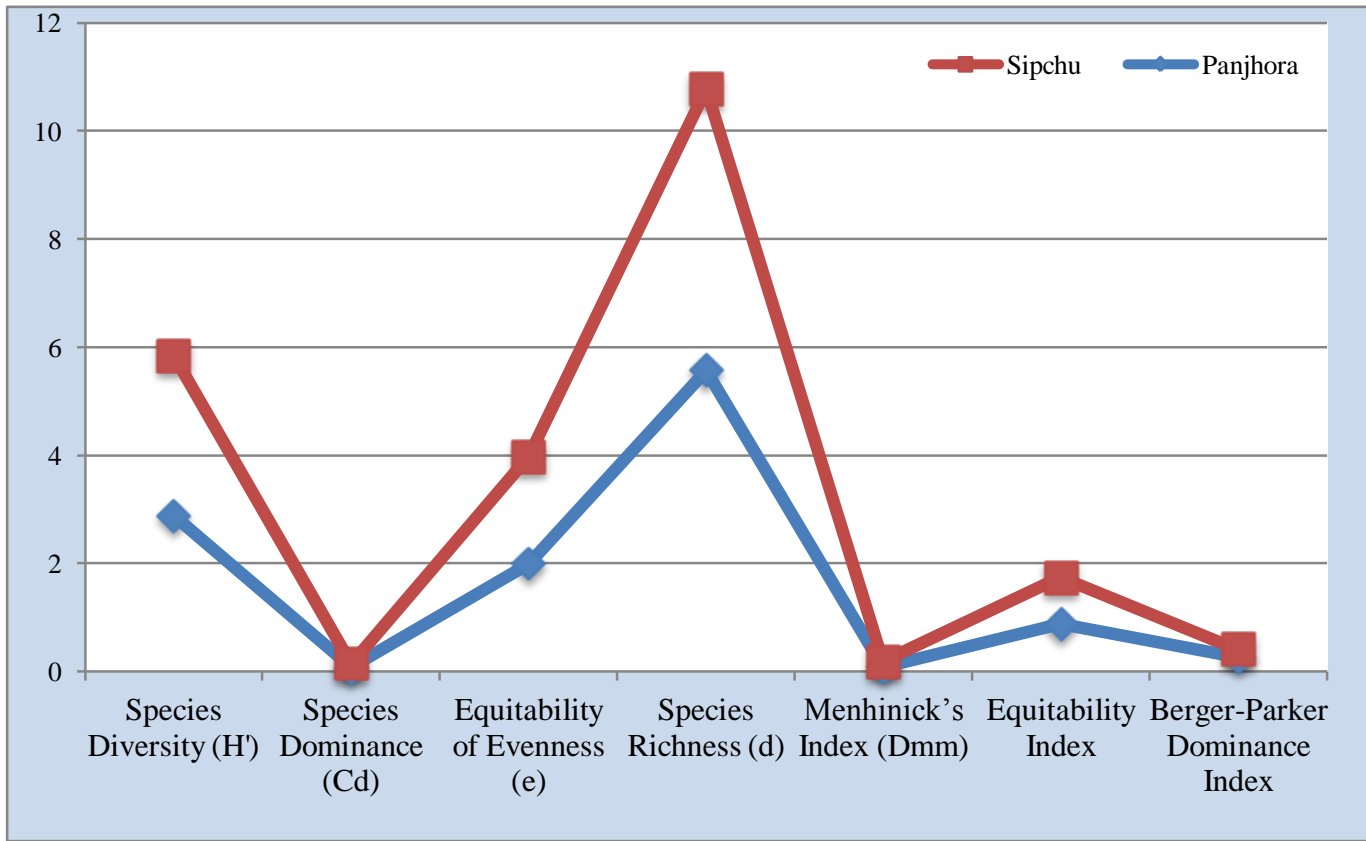

Graph 3: Community indices of tree vegetation of the Forest Beats. 


\section{ACKNOWLEDGEMENTS}

We convey our thanks and gratitude to Principal Chief Conservator of Forest, Chief Conservator of Forest and Divisional Forest Officer for their kind permission. We also great full to Dr. Archan Bhattacharya for his unreserved professional advice and encouragement. We also convey our thanks to Mr. Pallab Mukherjee, Range officer, Chalsa Range and his staffs for their kind cooperation during field study.

\section{Financial support and sponsorship: Nil.}

Conflict of Interests: There are no conflicts of interest.

\section{REFERENCES}

1. Battles JJ, Shlinsky AJ, Barrett RH, Heald RC and Allen-Diaz BH. The effects of forest management on plant species diversity in a Sierran conifer forest. Forest Ecology and Management, 2001;146: 211-222.

2. Berger WH and Parker FL. Diversity of plank tonic Foraminifer in deep sea Sediments Science, 1970;168:1345-1347.

3. Chaubey OP, Prasad R and Mishra GP. Studies of Teak Plantation and mixed natural forest in Madhya Pradesh. J. Trop. For. 1988;4: 22-35.

4. Chase JM and Leibold MA. 2003; Ecological Niches: Linking Classical and Contemporary Approaches. University of Chicago Press, Chicago.

5. Das D, Pramanik BK and Mollay SK. A Model Classical Ecological report on Vegetation dynamics in Gorumara National Park in West Bengal, India. Int. J. Phar. \&Biomedi. Rese., 2015;2(2):1-14.

6. Hunter MD. Differential susceptibility to variable plant phenology and its role in competition between two herbivores on Oak. Ecological Entomology. 1990; 12: 373-382.

7. Khosla PK. 1992.Staus of Indian forestry problem and perspective. Indian Society of Tree Scientists, University of Horticulture and Forestry, Solan (H.P.) India.

8. Kumar A and Ram J. Anthropogenic disturbances and plant biodiversity in forests of Uttaranchal, Central Himalaya. Biodiversity and Conservation, 2005; 14(2): 309-331.

9. Lloyd $\mathrm{M}$ and Ghelard RJ.A table for calculating the equitability component of species diversity, Journal of Animal Ecology, 1964; 33: 217-255.
10. Margalef R. 1968; Perspective in Ecological Theory, Uni. Of Chicago Press, 112.

11. Menhinick's Index as Described by Whittaker, R. H. Evolution of species diversity In land communities. In Evolutionary biology eds Heeht M. K., W. C. Steee and B Wallace Plenum, NY. 1977.

12. Osorio LF, Bravo F, Zaldiver P and Pando V. Forest structure and plant diversity in aritime pine Pinus pinaster (Ait) stands. in central Spain. Investigación Agraria. Sistemas Recursos Forestales, 2009; 18(3):314-321

13. Phillips EA. Methods of vegetation study. Henri Holt Co. Inc. 1959.

14. Pielou, EC. Species Diversity and pattern diversity of in the study of Ecological; Succession, Jour. Of theoretical Biology, 1966;10: 370383.

15. Sarkar AK. Ecological Studies of Tree Vegetation of Ramshai Forest Range, Gorumara National Park, India. Research Journal of Biological Sciences 2016; 5(7):53-59.

16. Sarkar AK. Phytosociological studies of tree vegetation of Moraghat forest range, India. Asian J. Biol. Life Sci. 2015;4(3):217-220.

17. Shannon CE and Wiener W.1963; The Mathematical theory of Communication, University Illinois Press, Urban.

18. Simpson, EH. Measurement of Diversity, Nature., 1949; 163: 688.

19. Uma Shankar. A case of high tree diversity in a sal (Shorea robusta)dominated lowland forest of Eastern Himalaya: Floristic composition, regeneration and conservation. Current Science, 2001;VOL.81, NO.7 (10): 776-786.

20. Vockenhuber EA, Scherber C, Langenbruch C, Meibbner M, Seidel $\mathrm{D}$ and Tscharntke $\mathrm{T}$. Tree diversity and environmental context predict herb species richness and cover in Germany's largest connected deciduous forest. - Perspectives in Plant Ecology, Evolution Systematics, 2011; 21(3): 111-119.

\section{How to cite this article:}

Sarkar AK, Dey M, Mazumder M. A Comparative Study of Tree Species Composition of Panjhora Forest Beat and Sipchu Forest Beat of Chalsa Forest Range,West Bengal,India. J App Biol Biotech. 2017; 5 (02): 045-052. DOI: 10.7324/JABB.2017.50207 